FORMATION Formation emploi

Revue française de sciences sociales

102 | avril-juin 2008

Pêle-mêle Présentation de l'ouvrage « Former pour réformer.
Retour sur la formation permanente (1945-2004)»

\title{
Marcelle Stroobants
}

\section{(2) OpenEdition}

1 Journals

Édition électronique

URL : https://journals.openedition.org/formationemploi/2316

DOI : 10.4000/formationemploi.2316

ISSN : 2107-0946

Éditeur

La Documentation française

Édition imprimée

Date de publication : 1 juin 2008

Pagination : 91-94

ISSN : 0759-6340

\section{Référence électronique}

Marcelle Stroobants, «Présentation de l'ouvrage « Former pour réformer. Retour sur la formation permanente (1945-2004) » », Formation emploi [En ligne], 102 | avril-juin 2008, mis en ligne le 02 décembre 2009, consulté le 21 septembre 2021. URL : http://journals.openedition.org/

formationemploi/2316 ; DOI : https://doi.org/10.4000/formationemploi.2316

(c) Tous droits réservés 


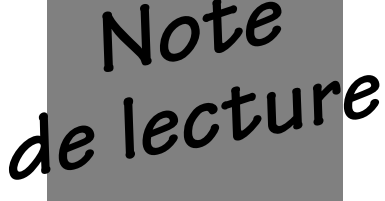

\section{Présentation de l'ouvrage "Former pour réformer. Retour sur la formation permanente (1945-2004)»}

Par Marcelle Stroobants*

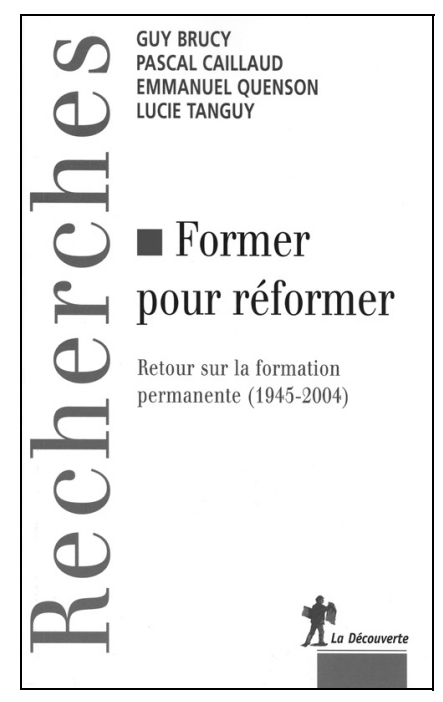

Il ne faut pas être archiviste pour deviner que l'injonction généralisée à la formation tout au long de la vie n'a pas grandchose à voir avec les idéaux de l'éducation permanente. S'il y a bien eu un détournement de la tradition, il ne se situe cependant pas là où on l'attendait; il remonte à plus d'une trentaine d'années. Encore fallait-il, pour s'en apercevoir, dépasser les définitions institutionnelles, les calendriers législatifs, les apparences consensuelles, reconstituer les conflits, les expériences - avec leurs tâtonnements - éplucher les documents de première main et faire parler les témoins de première ligne. Les résultats sont aussi de première qualité, offerts par une équipe de deux sociologues - Lucie Tanguy et Emmanuel Quenson - un historien - Guy Brucy - et un juriste - Pascal Caillaud - au terme d'une recherche très approfondie.
La «sociohistoire » qu'ils nous proposent couvre un demi-siècle, remontant aux circonstances tendues de l'après-guerre, moment où, rappelle Brucy, l'impératif productiviste rimait moins avec la rationalisation qu'avec la mobilisation du facteur humain et la polyvalence... déjà ! Dès le début des années 60 , reconversion et mobilité entrent en résonance avec les aspirations des cadres, des employés et le sort des agriculteurs. Quant aux ouvriers de cette génération, pour qui l'ascension professionnelle est peu probable, ils nourrissent une grande méfiance à

* Marcelle Stroobants est sociologue du travail et titulaire de plusieurs cours (sociologie du travail, de l'emploi, des professions, etc.) à l'université libre de Bruxelles. Elle a récemment publié : Sociologie du Travail, nouvelle édition refondue, Paris, Armand Colin, 2007. "Postface : la fabrication des compétences, un processus piloté par l'aval ? ", dossier Formation Emploi, n99, 2007, pp. 89-94. "L'individualisation des relations salariales par la gestion des compétences », in Dupray A., Guitton C., Monchatre S. (Coord.), Réfléchir la compétence. Approches sociologiques, juridiques, économiques d'une pratique gestionnaire, Toulouse, Octares, 2003, pp. 43-52. 
l'égard des méthodes américaines fraîchement importées dans les entreprises.

Fallait-il changer l'École pour ou par la formation permanente ? C'est la seconde voie qui allait être empruntée, moyennant une redistribution de leurs tâches respectives. Sous couleur de «seconde chance », la formation des adultes se démarque, dès la loi de 1971, de l'esprit - sinon de la lettre - d'une éducation populaire, largement orientée vers la culture et la citoyenneté.

Lucie Tanguy interroge d'abord la manière dont la formation, une fois présentée comme un «bien universel », a pu supplanter l'éducation, comment le système scolaire a pu être instrumentalisé et l'enseignement supérieur professionnalisé, suivant les orientations longuement préconisées par l'OCDE (Organisation de coopération et de développement économiques). Les glissements sémantiques ne sont jamais neutres, «autres mots, autres politiques», insiste Tanguy. Alors que «l'éducation » trouve aisément sa raison d'être en elle-même, la «formation » va chercher de multiples justifications dans les finalités économiques - même ambivalentes - qu'elle pourrait remplir. Les objectifs productivistes, de mobilité professionnelle, de promotion et de pacification sociale ont ébranlé puis éclipsé les orientations de l'éducation populaire.

Loin d'être une conquête du mouvement ouvrier, la formation continue s'avère résulter des actions d'élites, d'experts et de militants chrétiens animés par des soucis de réforme sociale et d'adaptation individuelle. Certains «missionnaires» de cette formation adoptaient d'ailleurs des termes assez misérabilistes pour décrire les bénéficiaires, définis par leurs manques, dépossédés, "démunis ». On découvre aussi, à cette occasion, que les pionniers ont mis en place des outils et des références conceptuelles étonnamment proches des formules actuelles : compétences, pédagogies actives, modules d'apprentissage et autres objectifs. Est-ce à dire que ces militants ont été "précurseurs de la pensée managériale qui s'est répandue deux décennies plus tard» (p. 47) ? Encore faudrait-il éprouver le corollaire de l'hypothèse et se demander si les mêmes mots recouvrent bien les mêmes politiques. Or, l'importance accordée aux contenus et aux collectifs dans les expériences pilotées par B. Schwartz ou M. David, par exemple, fait d'abord toute la différence avec les initiatives des entreprises. La suite de l'analyse confirme, en outre, que la pédagogie et la méthodologie, inhérentes au modèle actuel des compétences, s'inscrivent dans une perspective de rationalisation, au service de «l'économie de la connaissance». Sous couleur de scientificité, le formalisme des procédures d'évaluation généralisées contribue à naturaliser la relation formation-emploi et à occulter les inégalités sociales converties en responsabilités individuelles.

En revanche, s'il y a bien une éducation ouvrière continue qui émane d'abord de ses propres forces et vise le changement social, c'est plutôt la formation syndicale. C'est encore à l'initiative d'intellectuels chrétiens que les Instituts du travail ont ainsi été accueillis, dès 1955, au sein des universités, dans une perspective de promotion sociale collective et à l'aide d'une pédagogie réinventée par opposition aux réflexes scolaires. Les matières sont sélectionnées par les syndicalistes qui, précise Tanguy, "savent ce qu'ils ne savent pas et qu'ils devraient savoir», élaborées avec les universitaires et constituées en savoir collectif dans des groupes composites de salariés et même d'artistes. Ce projet initial s'est quelque peu dispersé avec l'institutionnalisation des syndicats et dans le mouvement général de professionnalisation.

Les diverses positions des syndicats sur la formation, étudiées par Guy Brucy, se montrent à la mesure des doctrines qui les opposent. CFTC (Confédération française des travailleurs chrétiens) et CGT-FO (Confédération générale des travailleurs - Force ouvrière) s'inscrivent en quelque sorte dans la filiation de Pelloutier - la "science de son malheur", c'est ce qui manque à l'ouvrier - voilà donc ce qu'il lui faut acquérir pour devenir un militant et un négociateur. De son côté, la CGT ne fait confiance qu'à elle-même pour entraîner des combattants à la lutte des classes. En ce qui concerne la formation professionnelle des ouvriers, les positions sont aussi divergentes; les uns en font un outil de co-gestion et de promotion, les autres dénoncent les tentatives de division et de «catéchisation » de la classe ouvrière par les méthodes des « relations humaines ». À partir des années 60 , ce sont les cadres qui, par-delà leurs affiliations syndicales, vont se faire idéologues d'un 
droit individuel à une nécessaire formation pendant le travail et sans perte de salaire, préfigurant une partie des principes de la loi de 1971 .

Emmanuel Quenson examine ensuite le dispositif d'information qu'il a fallu mettre en place pour convaincre les salariés d'utiliser ce fameux congéformation auquel cette loi leur donnait droit. Le CNIPE (Centre national d'information pour le progrès économique) devait ainsi propager, avant la lettre, cette figure du salarié acteur - responsable de la construction de sa qualification et de sa carrière mobilisé pour la formation perpétuelle.

Pascal Caillaud analyse le déplacement par lequel le droit de la formation des adultes s'est progressivement émancipé de l'État pour s'orienter vers les initiatives privées et s'aligner sur des normes du contrat de travail. La gestion paritaire de ce système a conféré plus de pouvoir aux partenaires sociaux du privé, à l'échelle régionale, au détriment des syndicats enseignants. Le droit collectif est désormais devenu individuel - diversifié selon le statut associé au «parcours » personnel - à la fois plus risqué et plus contraignant pour le principal intéressé. À présent, estime Caillaud, le modèle consumériste semble prendre le pas sur le droit du travail. Incité à investir du temps personnel dans sa formation, le salarié voit désormais son statut assimilé à celui d'un co-contractant, au risque de perdre le bénéfice des dispositions $\mathrm{du}$ code du travail qui compensent l'inégalité entre salarié et employeur.

Qu'en était-il alors de la formation des adultes avant cette fameuse loi de 1971 ? L'Éducation nationale, si prégnante en France, n'y a-t-elle joué que ce rôle mineur qu'on lui attribue d'ordinaire rétrospectivement? En historien, Guy Brucy ne peut se satisfaire d'un récit finalisé par la voix des vainqueurs et repart sur les traces du « perfectionnement » professionnel. Car c'est ce terme qui, de 1920 à 1970, désigne les cours du soir organisés dans les établissements techniques à destination des adultes. Conçu dans l'entre-deux-guerres comme une articulation de la formation, de la certification et de la promotion - " antidote à la lutte des classes » - ce dispositif s'est constitué sur un pacte entre employeurs et enseignants. Les premiers gardaient la maîtrise de l'organisation du travail tout en participant aux coûts de la formation ; les seconds ne pouvaient que contri- buer au "perfectionnement», sans illusion sur sa reconnaissance par les premiers. Cette tension, aiguisée dans un rapport salarial à la française ${ }^{1}$, allait progressivement déstabiliser ce compromis de part et d'autre. La fréquentation des cours, le soir et une grande partie du week-end représente en effet, pour la plupart des travailleurs, un exploit d'autant plus exorbitant que sa contrepartie reste incertaine.

Depuis les «confrontations» entre des « interlocuteurs » sociaux autonomes jusqu'au «dialogue» social, en passant par des «partenaires » sociaux, beaucoup de termes équivoques ont progressivement édulcoré les relations sociales. Quelle peut-être la portée de ce dialogue social instauré dans cette loi sur la formation tout au long de la vie ? Lucie Tanguy s'atèle à la délimiter, dans un dernier chapitre qui parachève le bouleversement des idées. Car contrairement à ce qu'on a pu croire, la loi de 1971 ne résultait pas des accords antérieurs, mais a été promue - sous la houlette de Jacques Delors - en vue d'impulser la concertation. Les deux parties se montraient, en fait, plus intéressées par la formation initiale, peu demandeuses de formation continue et n'avaient d'ailleurs pas développé d'expertise pointue en ce domaine, considéré comme « un art majeur ». Le syndicalisme affaibli, institutionnalisé, est resté prioritairement mobilisé sur les questions d'emploi au détriment de la formation. De la sorte, le paritarisme gestionnaire ne laisse aujourd'hui guère de place à sa part syndicale. La formation, "laboratoire d'expérimentations sociales et politiques » conclut Tanguy, révèle ainsi toute sa dimension politique.

Au moment de célébrer le quarantenaire de mai 68, on se plaira à rappeler que, pour meubler les fameux accords de Grenelle - dont le vide avait frappé Pompidou - il fallut y injecter, en dernière minute, « une petite phrase sur la formation »!

Bien au-delà des mots, des généalogies, chronologies et autres continuités sur lesquelles les auteurs insistent, les contrastes, mais aussi les tensions réactivées ici appellent à poursuivre cette pénétrante « sociohistoire ».

\footnotetext{
${ }^{1}$ Par comparaison à d'autres pays où les relations collectives ont pu s'organiser dans le cadre du métier, le rapport salarial est, en France, traditionnellement marqué par la dispersion syndicale, la prégnance du système éducatif et des qualifications peu transférables.
} 


\section{Référence de l'ouvrage}

Guy Brucy, Pascal Caillaud, Emmanuel Quenson, Lucie Tanguy (2007), Former pour réformer. Retour sur la formation permanente (1945-2004), Paris, La Découverte. 